\title{
Clinicopathological Features of Adrenal Tumors: a Ten-year Study in Yazd, Iran
}

\author{
Shokouh Taghipour Zahir ${ }^{1 *}$, Ezatollah Aalipour ${ }^{2}$, Poorya Barand ${ }^{2}$, Mansoureh \\ Kaboodsaz $^{3}$
}

\begin{abstract}
Background: Adrenal tumors are relatively uncommon, and have different presentations, so we decided to evaluate the clinico-pathological characteristics of benign and malignant tumors in a ten-year period. Materials and Methods: This cross sectional-analytical study was conducted on adrenal resection samples taken during 2004-2014 in three hospitals in Yazd province. Data were analyzed using SPSS software, version 17. Chi-square and Fisher's exact test were used as appropriate Results: A total of 71 patients with adrenal tumors were analyzed, including $32(45.1 \%)$ men and $39(54.9 \%)$ women with an overall mean age $37.7 \pm 19.9$ (range: 6-75 years). Some $50.7 \%$ of lesions were benign and $49.3 \%$ were malignant. Neuroblastoma was the most malignant lesion $(32.3 \%)$ followed by adrenocortical carcinoma $(8.4 \%)$. Among the benign lesions pheochromocytoma was the most common (25.3\%) followed by adrenocortical adenoma (12.6\%). While $64 \%$ of tumors were functional $36 \%$ were non functional. Significant correlation was seen between the age of patient and type of tumor $(P=0.001)$. In patients between 14-40 years old no malignant lesions was found, although under 14 years old all of the tumors were malignant. Malignant lesions mostly presented with abdominal pain, abdominal mass and anorexia $(57.2 \%$, 45.7\% and $\mathbf{4 5 . 7 \% )}$ ) respectively. Benign lesions mostly presented with paroxysmal hypertension, headache and abdominal pain $(61.2 \%, 47.2 \%$ and $44.4 \%)$ respectively. Conclusions: Since the trend of adrenal tumors is on the rise based on this and other studies, suspected cases should undergo prompt hormonal and radiological assessment. Early diagnosis and treatment could prevent tumor progression and reduce mortality and morbidity rates.
\end{abstract}

Keywords: Adrenal tumors - malignancy - pathology - pheochromocytoma

Asian Pac J Cancer Prev, 16 (12), 5031-5036

\section{Introduction}

The adrenal glands are retroperitoneal organs located above the kidneys. Increased function of the adrenal cortex creates three main groups of clinical syndromes related to elevated hormone levels: Cushing's syndrome, hyperaldosteronism, and a number of androgenic syndromes. Hyperaldosteronism and Cushing's syndrome mostly accompany the functional adenomas of the adrenal and an androgenic neoplasm is more likely to be a carcinoma (Ciftci et al., 2001). Some adrenal masses do not have any hormonal function and present with abdominal mass or pain (Michalkiewicz et al., 2004). Determining the functionality of a cortical neoplasm is possible based on clinical evaluation and measuring the hormone or its metabolites in the laboratory. Differentiation is not possible with morphology characteristics per se (Michalkiewicz et al., 2004; Sadler C and Goldfarb., 2014). Most cortical adenomas are non-functional tumors and are often detected incidentally during autopsy or in abdominal radiography. Therefore, the term adrenal incidentaloma was used to describe such tumors (Li et al., 2014).These masses exist in about $2-3 \%$ of the general population and their incidence increases with age (1\% at age 40 and 7\% at age 70) (Pędziwiatr et al., 2014; Jing et al., 2014). Adrenal neoplasms range from asymptomatic cysts to fatal carcinomas. The most common benign adrenal tumors include pheochromocytoma, adrenocortical adenoma, myelolipoma, and simple cysts (Major et al., 2012). The most common malignant adrenal tumors are adrenocortical carcinoma, neuroblastoma, and metastasis from other organs malignant tumors (Major et al., 2012; Zang et al., 2014). Considering the variant pathological views of adrenal tumors and their varying clinical symptoms, we aimed to evaluate the clinicopathological features of adrenal tumors in a 10-year period in Yazd city, center of Iran, for the first time with respect to type, age, sex, side, clinical, pathological, and hormonal symptoms.

\section{Materials and Methods}

This cross -sectional study was done on all adrenal

${ }^{1}$ Pathology Medicine, ${ }^{2}$ Research committee,${ }^{3}$ Internal Medicine, Medical Faculty, Shahid Sadoughi University of Medical Sciences, Yazd, Iran*For correspondence: taghipour@ssu.ac.ir 
biopsy samples taken during 2004-2014 in three hospitals (Shahid Sadoughi, Mortaz, and Mojibian) in Yazd, that were diagnosed as benign or malignant. We used the census method to sampling and included all pathology records of patients with a confirmed diagnosis of benign or malignant adrenal tumor. Data were gathered using a questionnaire consisting of variables such as age, sex, clinical symptoms and anatomical side of the lesion, pathology findings, and the tumor's hormonal function. We excluded patients' records that were incomplete. All patients' data were approved for use by the institutional review board of Shahid Sadoughi University of medical science and services. All statistical analyses were performed with the computer program Statistical Package for Social Sciences (SPSS) version 17.0 for Windows (Chicago, Illinois). Fisher's exact and chi-square tests were used to compare the properties of the patients and pathological findings. $\mathrm{P}<0.05$ was considered as statistically significant.

\section{Results}

In this study, a total 71 patients were enrolled. Demographic characteristics of patients along with anatomical side of lesions are presented in Table 1. Women comprised $54.9 \%$ of the participants, and the mean age at diagnosis was $37.7 \pm 19.87$ (range: 6-75 years). Adrenal lesions were most frequently detected in patients less than 14 years of age and adults who were 41-50 years old. We found a significant difference between age and type of tumor ( $\mathrm{P}<0.001$, Chi-square test), so in patients over 40 years of age, the rate of malignancy increased with an increase in age. Moreover, all tumors in patients less than

Table 1. Baseline Characteristics of Patients and Adrenal Lesions

\begin{tabular}{llcc}
\hline Patients & Female & $\begin{array}{c}\text { Malignant } \\
(\mathrm{N}=35)\end{array}$ & $\begin{array}{c}\text { Benign } \\
(\mathrm{N}=36)\end{array}$ \\
\hline Gender, n (\%): & $\begin{array}{c}18(51.4) \\
\text { Male }\end{array}$ & $\begin{array}{c}21(58.3) \\
17(48.6)\end{array}$ \\
Age strata, n (\%): & $<14$ & 23 & 0 \\
& $14-30$ & 0 & 9 \\
& $31-40$ & 0 & 8 \\
& $41-50$ & 3 & 10 \\
& $51-60$ & 5 & 7 \\
Lesions: Anatomical origin of lesions, $\mathrm{n}(\%)$ & 2 \\
Left adrenal & $>60$ & $25(71.5)$ & $17(47.2)$ \\
Right adrenal & & $10(28.5)$ & $19(52.8)$ \\
\hline
\end{tabular}

14 years of age were malignant and all adrenal tumors in 14-40 year-old patients were benign. Final diagnosis of malignancy was made in 35 lesions (49.3\%), and the remainder (36 lesions, 50.7\%) was classified as benign. The most common anatomical side of lesions were left adrenal (42 lesions, 59.2\%), and then right side (29 lesions, $40.8 \%$ ) respectively.

\section{Benign lesions}

Most frequent types of benign lesions were pheochromocytoma (18 lesions, 25.3\%), and adrenocortical adenoma (9 lesions, 12.6\%) (Table2). Frequency of clinical signs and symptoms for benign adrenal lesions are presented in Table 3. The most common reported clinical presentation was paroxysmal hypertension (61.2\%), followed by headache (47.2\%) and abdominal pain $(44.4 \%)$. Comparision between benign and malignat lesions detected that, patients with benign lesions were significantly more likely to experience paroxysmal hypertension, followed by excessive sweating, headache and palpitation, but patients with malignant lesions had an abdominal pain $(57.2 \%)$ followed by abdominal mass, loss of appetite, weight loss and prolonged fever. Most benign adrenal tumors were on the right side $(52.7 \%)$ and most of the malignant tumors were on the left side (71.4\%), but there was no significant difference between the side and type of tumor ( $\mathrm{P}=0.84$ and $\mathrm{P}=0.39$ ) Respectively (Fisher's exact test). With respect to hormonal status, (69.4\%) of benign tumors were functional however between the benign lesions, patients with pheochromocytoma were significantly experience more hormonal functionality compared with others $(\mathrm{P}=0.001)$ (Table 4). In abdominal ultrasonography half of the benign tumors were more than $4 \mathrm{~cm}$ but between benign group no significant difference was found $(\mathrm{P}=0.54)$ (Table 2).

\section{Malignant lesions}

Pathological evaluation of available specimens showed that neuroblastoma and adrenocortical carcinoma are the two most common histological types of malignancy identified in $23(32.3 \%)$ and $6(8.4 \%)$ patients, respectively. In patients over 40 years of age, the rate of malignancy increased with an increase in age. Moreover, all tumors in patients less than 14 years of age were malignant. Frequency of clinical sign and symptoms for malignant lesions are presented in Table 3 . The most common reported clinical presentation was abdominal pain (57.2\%), followed by abdominal mass and anorexia (each one $45.7 \%$ ). Most of the malignant tumors had a size more

Table 2. The frequency (\%) Distribution of Malignant and Benign Adrenal Tumors Based on Side and Size

\begin{tabular}{|c|c|c|c|c|c|c|}
\hline Type & & $\begin{array}{l}<4 \mathrm{~cm} \\
\mathrm{~N} \%\end{array}$ & $\begin{array}{l}>4 \mathrm{~cm} \\
\mathrm{~N} \%\end{array}$ & $\begin{array}{r}\text { Right } \\
\mathrm{N} \%\end{array}$ & $\begin{array}{l}\text { Left } \\
\mathrm{N} \%\end{array}$ & $\begin{array}{l}\text { Total } \\
\mathrm{N} \%\end{array}$ \\
\hline \multirow[t]{4}{*}{ Malignant } & Neuroblastoma & $8(34.7)$ & $15(65.3)$ & $8(34.8)$ & $15(65.2)$ & $23(100)$ \\
\hline & Adrenocortical Carcinoma & $0(0)$ & $6(100)$ & $1(16.6)$ & $5(83.3)$ & $6(100)$ \\
\hline & Pheochromocytoma & $0(0)$ & $4(100)$ & $1(25)$ & $3(75)$ & $4(100)$ \\
\hline & Metastasis & $0(0)$ & $2(100)$ & $0(0)$ & $2(100)$ & $2(100)$ \\
\hline \multirow[t]{4}{*}{ Benign } & Pheochromocytoma & $10(55.5)$ & $8(44)$ & $9(50)$ & $9(50)$ & $18(100)$ \\
\hline & Adrenocortical Adenoma & $5(55.5)$ & $4(44.5)$ & $3(33.4)$ & $6(66.6)$ & $9(100)$ \\
\hline & Cyst & $1(20)$ & $4(80)$ & $3(60)$ & $2(40)$ & $5(100)$ \\
\hline & Myelolipoma & $2(50)$ & $2(50)$ & $4(100)$ & $0(0)$ & $4(100)$ \\
\hline
\end{tabular}


Table 3. Frequency (\%) of Clinical Signs and Symptoms in Benign and Malignant Lesions

\begin{tabular}{lrrl}
\hline & $\begin{array}{c}\text { Malignant } \\
(\mathrm{n}=35)\end{array}$ & $\begin{array}{c}\text { Benign } \\
(\mathrm{n}=36)\end{array}$ & Pvalue \\
& $\mathrm{N}(\%)$ & $\mathrm{N}(\%)$ & \\
\hline Abdominal pain & $20(57.2)$ & $16(44.4)$ & 0.35 \\
Paroxysmal hypertension & $9(25.7)$ & $22(61.2)$ & 0.004 \\
Incidental finding & $13(37.2)$ & $14(38.8)$ & 1 \\
Abdominal mass & $16(45.7)$ & $9(25)$ & 0.08 \\
Headache & $3(8.8)$ & $17(47.2)$ & $<0.001$ \\
Nausea and Vomiting & $9(25.7)$ & $11(30.5)$ & 0.8 \\
Anorexia & $16(45.7)$ & $4(11.1)$ & 0.001 \\
Palpitation & $4(11.4)$ & $15(41.6)$ & 0.007 \\
Weakness & $9(25.7)$ & $10(27.7)$ & 1 \\
Weight loss & $15(42.8)$ & $4(11.1)$ & 0.003 \\
Excessive sweating & $4(11.4)$ & $13(36.1)$ & 0.025 \\
Prolonged fever & $12(34.2)$ & $0(0)$ & 0.001 \\
Weight gain & $0(0)$ & $4(11.1)$ & 0.36 \\
Hirsutism & $0(0)$ & $4(11.1)$ & 0.36 \\
\hline
\end{tabular}

Table 4. The Frequency (\%) Distribution of Malignant and Benign Adrenal Tumors Based on Hormonal Function

\begin{tabular}{lccc}
\hline Type & Functional & Non-functional \\
& $\mathrm{N} \%$ & $\mathrm{~N} \%$ & P value \\
\hline Malignant & & & \\
Neuroblastoma & $13(56.5)$ & $10(43.5)$ & \\
Adrenocortical Carcinoma & $4(66.6)$ & $2(33.4)$ & \\
Pheochromocytoma & $4(100)$ & $0(0)$ & 0.59 \\
Metastasis & $0(0)$ & $2(100)$ & \\
Benign & & & \\
Pheochromocytoma & $18(100)$ & $0(0)$ & \\
Adrenocortical Adenoma & $5(55.5)$ & $4(44.5)$ & 0.001 \\
Cyst & $1(20)$ & $4(80)$ & \\
Myelolipoma & $1(25)$ & $3(75)$ & \\
\hline
\end{tabular}

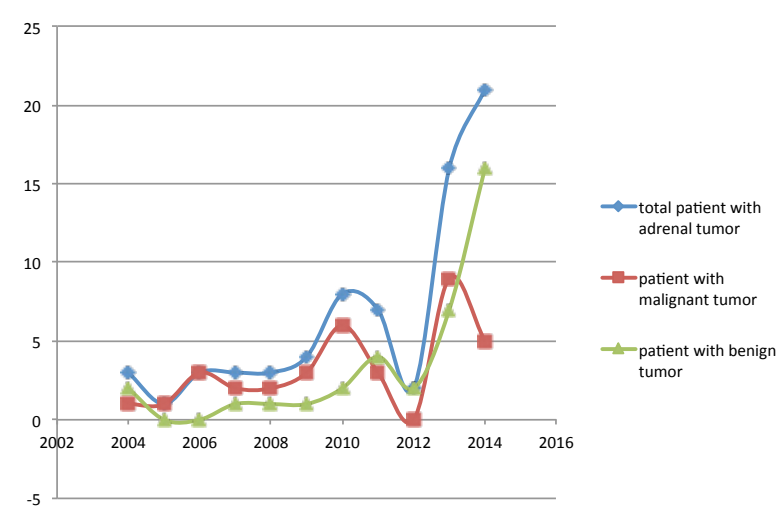

Figure 1. The Trend of Adrenal Lesions During 20042014

than $4 \mathrm{~cm}$, but no significant difference was seen between the type of tumors and their size $(\mathrm{P}=0.72)$. With regard to functional status of malignant tumors, although most of the malignant tumors were functional, but no significant difference was seen between them $(\mathrm{P}=0.59)$.

\section{Incidence and trends of adrenal tumors}

The incidence of adrenal masses was on the rise during the 10 -year study period, from 3 in 2004 to 21 in 2014. The
Clinicopathological Assessment of Adrenal Tumors in Yazd, Iran incidence of benign masses had risen from 2 in 2004 to 16 in 2014, and the incidence of malignant masses has also increased from 1 in 2004 to 9 in 2013, while decreasing to 5 in 2014 (figure 1).

\section{Discussion}

In an attempt to fill the available knowledge gap in epidemiology of adrenal tumors in Iran, we reported clinicopathological features of adrenal tumors in Yazd between 2004 and 2014. Based on our observations, annual incidence of adrenal malignancies had gradually and consistently increased over the past decade. Adrenocortical neoplasms composed of adrenocortical carcinoma and adrenocortical adenoma mostly occur in adults, however pediatrics are not being safe from it, and few records have been published about pediatrics adrenocortical tumors (Ciftci, 2001). In our study benign tumors were mostly seen in adults between age 41-50 years old, followed by 51-60 years old and our findings are consistant with other studies (Li et al., 2014; Pędziwiatr et al., 2014; Jing et al., 2014). Adrenocortical carcinoma is rare in pediatrics and in our study we had not any adrenocortical carcinoma among the ages less than 41 years old, however, (Ciftci et al., 2001) reported adrenocortical carcinoma in children less than 13 years old. So our study was different from other studies. Adrenocortical neoplasms have an equal sex distribution, although our study demonstrated that the frequency of adrenal masses were slightly higher in women than men, but this difference was not statistically meaningful and our findings were consistent with previous studies which have been published by cancer statistics of USA (Anon et al., 2015). Adrenocortical neoplasms are presented as hormonal dysfunction or may be evident by mass effect (Kerkhofs et al., 2013; Zhang and Liu, 2008). In our study the most frequent clinical symptoms of patients with adrenocortical carcinoma were abdominal pain followed by weight loss. Our results differ from other studies in which other symptoms such as abdominal mass, Cushing's syndrome, increased steroidal hormones and endocrine symptoms were more commonly observed in patients with adrenocortical carcinoma (Kerkhofs et al., 2013; Zhang, 2008; Icard et al., 2001; Stojadinovic et al., 2001). In patients with adrenocortical adenoma most clinical symptoms were paroxysmal hypertension followed by abdominal pain (2008; Icard et al., 2001; Stojadinovic et al., 2001). Fassnacht et al. also reported that patients' clinical signs and symptoms along with hormonal status had a limited predictive value and for differentiation benign and malignant tumors from each other imaging studies along with histopathological findings should be used (Fassnacht et al., 2011). In our study most adrenocortical adenomas were functional (55.5\%), which is in line with the previously mentioned study but inconsistent with Mantero and colleagues' study (Mantero et al., 2000). Moreover, most adrenocortical carcinomas were also functional (66.6\%) in contrast to Ayala-Ramirez $\mathrm{M}$ et al. study that non-functional carcinomas (58.2\%) were more than functional ones $(41.8 \%$ ) (Ayala-Ramirez et al., 2013). Differential diagnosis between adrenocortical 
adenoma and carcinoma, and in some cases even between primary and secondary adrenal tumor, still remains the challenge for pathologist, although applying of immunohistochemistry and Weiss criteria may be helpful (Fassnacht et al., 2011). The number of functional adrenocortical tumors may actually be higher than reported because some nonfunctional tumors later become functional. Therefore, it may ordinary that studies have reported the prevalence of the functional tumors higher than its nonfunctional type (Icard et al., 2001; Fassnacht et al., 2011).In our study the average age for adrenocortical carcinoma was about 50 years with male predominance $(66.7 \%)$ compare with female (33.3\%), but this difference was not statistically significant. Our results were inconsistent with other similar studies, which reported the higher prevalence of adrenocortical carcinoma in female (Ayala-Ramirez et al., 2013) and inline with Keskin S et al. study (Keskin et al., 2013). Also in present study, the frequency of adrenocortical adenomas was higher in females, which was consistant with Audenet et al study (Audenet et al., 2013). As compared to other studies the mean age of our patients with adrenocortical adenoma was 38.7 years and this finding indicates that patients with adrenocortical adenoma were one to two decate younger than patients with adrenocortical carcinoma. Neuroblastoma is the most common extracranial solid tumor of childhood. It commonly presents in children younger than 2 years of age, with $90 \%$ being younger than 5 years of age (Missaoui et al., 2011; Mehdiabadi et al., 2013). In present study all the patients with neuroblastoma were younger than 14 years old and also there was a very minimal difference between the frequency of neuroblastoma in males and females (11 female, 12 male). In reported cases it is slightly more common in males than females (Gao et al., 2007). Mehdiabadi et al. reported the male/ female ratio of neuroblastoma about $1.9: 1$, which was approximately in accordance with our study (Mehdiabadi et al., 2013). We observed that in patients with neuroblastoma, the most commonly clinical symptom was abdominal mass followed by abdominal pain. This finding is consistent with previous reports (Gao et al., 2007; Mehdiabadi et al., 2013). Pheochromocytoma, an adrenal medulla paraganglioma, has been known as $10 \%$ tumor, which is seen $10 \%$ bilaterally, $10 \%$ in children and $10 \%$ malignant. Surprisingly, in this study $75 \%$ of all malignant and half of benign cases of pheochromocytomas were in the left side. This is in contrast to other series of studies in the literature, which showed that the most cases of the pheochromocytomas tend to be right side (Fitzgerald et al., 2006). Our research showed that benign pheochromocytoma was higher in the 14-30 and 41-50 year-old age groups. Between benign adrenal tumors pheochromocytoma was the most common tumor arise from adrenal medulla and our findings were aline with previous published studies ((Fitzgerald et al., 2006; Gokuldas and Selvaraju, 2013). Nearly pheochromocytomas comprised $25 \%$ and $5.7 \%$ of all benign and malignant adrenal tumors, respectively. Its male to female ratio among benign cases was same, while the proportion was $3: 1$ for malignant cases and female patients definitely predominant. The last finding was unlike previous studies, which reported its higher incidence among males (Jiang et al., 2011). But another study also mentioned that male to female ratio in benign pheochromocytoa was the same (Ioannis and Karel, 2008). Among the secondary causes of hypertension, pheochromocytoma is a curable form of hypertension and reported in $0.1-1.0 \%$ of patients with hypertension (Omura et al., 2004). Despite its role in hypertension, it is not routinely screen for every patient with hypertension. Usually the symptoms occur paroxysmally, but approximately $50 \%$ of the patients have sustained hypertension. Accordingly, it seems that pheochromocytoma is a very highly heterogeneous disease. Similarly in our study among both benign and malignant pheochromocytoma cases paroxysmal hypertension, abdominal pain, palpitation and excessive sweating were common in all. These findings were comparable to the values reported by (Gokuldas Shenoy and Selvaraju, 2013). Adrenal cysts are uncommon adrenal tumor like lesions and clinically can be confused with other retroperitoneal lesions. In our study adrenal cysts were more frequent in female than male, and the patients age were more than 14 years old. Also $80 \%$ of cysts had a size more than $4 \mathrm{~cm}$. These findings are in line with other studies (Lyu et al., 2014). In our study all of the cysts were incidentally identified. By increasing the use of computed tomography scan and ultrasound it seems that incidentaly identification of adrenal mass like lesions are increased (Kanagarajah et al., 2012; Sebastiano et al., 2013; Degenhart, 2014). Myelolipoma is a rare adrenal benign tumor that is often discovered accidentally, and composed of fatty tissue containing active bone marrow elements (Bezjak et al., 2013). In our study it was similarly found in men and women and its frequency did not differ across the sexes. The main clinical symptoms were abdominal pain and weakness (50\% each one). In reported cases it was rare in children and similarly in our study no myelolipoma was found in patients under 30 years old. All of our myelolipomas were in left side, and $75 \%$ of them were non functional. With respect to rarity of myelolipoma, the most common finding was an incidentally detected mass, however in our study $50 \%$ of patients had an abdominal pain (Treska et al., 2006). In our study the size of most adrenal tumors was more than $4 \mathrm{~cm}$, which is similar with previous report (Keskin et al., 2013). We found a significant difference between the size of benign and malignant tumors. Therefore, size of tumor could be used as a reliable marker for differentiation benign tumors from malignant ones. This finding is consistent with previous studies (Jiang et al., 2011). The size of the tumor is of importance, adrenal masses $5 \mathrm{~cm}$ or more in diameter are frequently but not invariably malignant, and the larger the mass, the more likely it is to be malignant (Jiang et al., 2011). However, most of the adrenocortical adenomas are less than $2 \mathrm{~cm}$ in greatest dimension. Neverthless, size and weight of the adrenal cortical tumors are no longer considered to be a reliable sign of benignity or malignancy. During the 10 years of our evaluation, the frequency of adrenal tumors had increased from 3 in 2004 to 21 in 2014. The frequency of benign tumors had increased from 2 in 2004 to 16 in 2014. We do not know whether this increased prevalence is due 
to the increased incidence of this disease in recent years or the increased use of imaging modalities for nonspecific complaints, which could incidentally detect asymptomatic adrenal tumors that are often benign. This finding is consistent with other studies (Degenhart, 2014; O'Neill et al., 2010). The frequency of malignant adrenal tumors has increased from 1 case in 2004 to 9 in 2013. However, in 2014, only 5 cases were reported. This finding is similar to a report by Fassancht who found that the prevalence of carcinoma had increased by over 15\% (Fassnacht et al., 2011). Considering the incidence of adrenal tumors and their non-specific symptoms and since all adrenal carcinomas are life threatening and that the size of nearly all adrenocortical carcinomas were more than $4 \mathrm{~cm}$ at diagnosis in our study, they should be diagnosed promptly. Also, since increase in the size of adrenal masses increases the possibility of malignancy, adrenal masses that are more than $4 \mathrm{~cm}$ should be excised in order to prevent local invasion and distant metastasis.

In conclusion, In this study the most prevalent benign and malignant lesions were pheochromocytoma and neuroblastoma, respectively. The incidence of adrenal masses was similar across the sexes. Also, the incidence of malignant and benign tumors was nearly the same. Since the trend of adrenal tumors is on the rise based on this study and other studies, suspected cases should undergo prompt hormonal and radiological assessment. Early diagnosis and treatment could prevent tumor progression and reduce mortality and morbidity rates.

\section{Acknowledgements}

The authors would like to thank Prof. dr. Karel Geboes, Prof. dr. Wim Ceelen.

\section{References}

Anon, (2015). [online] Available at: http://American Society of Clinical Oncology (ASCO)

Audenet F, Méjean A, Chartier-Kastler E, Rouprêt M (2013). Adrenal tumours are more predominant in females regardless of their histological subtype: a review. World J Urol, 31, 1037-43.

Ayala-Ramirez M, Jasim S, Feng L, et al ( 2013). Adrenocortical carcinoma: clinical outcomes and prognosis of 330 patients at a tertiary care center. Eur J Endocrinol, 169, 891-9

Bezjak M, Sesar P, Ulamec M, et al (2013). [Adrenal myelolipoma--report of 15 patients]. Acta Med Croatica, 67, 255-8.

Ciftci AO, Senocak ME, Tanyel FC, Buyukpamukcu N (2001). Adrenocortical tumors in children. J Pediatr Surg, 36, 549-54.

Degenhart C(2014). [Adrenal tumors: Principles of imaging and differential diagnostics]. Radiologe, 54, 998-1006.

Fassnacht M, Libé R, Kroiss M, et al (2011). Adrenocortical carcinoma: a clinician's update. Nat Rev Endocrinol, 7, 323.

Fitzgerald PA, Goldsby RE, Huberty JP, et al (2006). Malignant pheochromocytomas and paragangliomas: a phase II study of therapy with high-dose 131I-metaiodobenzylguanidine (131I-MIBG). Ann N Y Acad Sci, 1073, 465-90.

Gao XN, Tang SQ, Lin J (2007). Clinical features and prognosis of advanced neuroblastoma in children. Zhongguo Dang Dai Er Ke Za Zhi, 9, 351-4.
Clinicopathological Assessment of Adrenal Tumors in Yazd, Iran

Gokuldas Shenoy M, Selvaraju K (2013). Clinicopathological analysis of pheochromocytoma: a retrospective study. WebmedCentral ENDOCRINE SURGERY, 4.

Icard P, Goudet P, Charpenay C, et al (2001). Adrenocortical carcinomas: surgical trends and results of a 253-patient series from the French Association of Endocrine Surgeons study group. World J Surg, 25, 891.

Ilias I, Pacak K (2008).A clinical overview of pheochromocytoma/ paraganglioma and carcinoid tumors. Nucl Med Biol, 35.

Jiang CX, Zeng Z, Wang T, et al (2011). [Pheochromocytomas in adrenal medulla or extra-adrenal and multiple endocrine neoplasms:a clinicopathologic analysis of 181 cases]. Zhonghua Bing Li Xue Za Zhi, 40,762-6.

Kanagarajah P,Ayyathurai R, Manoharan M, Narayanan G, Kava BR (2012). Current concepts in the management of adrenal incidentalomas. Urol Ann, 4, 137-44

Kerkhofs TM, Verhoeven RH, Van der Zwan JM, Dieleman J, Kerstens MN (2013). Adrenocortical carcinoma: a population-based study on incidence and survival in the Netherlands since 1993. Eur J Cancer, 49, 2579-86.

Keskin S, Taş F, Vatansever S (2013). Adrenocortical carcinoma: clinicopathological features, prognostic factors and outcome. Urol Int, 90, 435-8.

Li L, Dou J, Gu W, et al (2014). [Clinical analysis of 4049 hospitalized cases of adrenal lesions]. Zhonghua Yi Xue Za Zhi, 94, 3314-8.

Lyu X, Liu L, Yang L, Gao L, Wei Q (2014). Surgical management of adrenal cysts: a single-institution experience. Int Braz J Urol, 40, 656-65.

Major P, Pędziwiatr M, Matłok M, et al (2012). Cystic adrenal lesions - analysis of indications and results of treatment. Pol Przegl Chir, 84, 184-9.

Mantero F, Terzolo M, Arnaldi G, et al (2000). A survey on adrenal incidentaloma in Italy. Study Group on Adrenal Tumors of the Italian Society of Endocrinology. J Clin Endocrinol Metab, 85, 637-44.

Mehdiabadi GB, Arab E, Rafsanjani KA, Ansari S, Moinzadeh AM (2013). Neuroblastoma in Iran: An Experience of 32 Years at a Referral Childrens Hospital. Asian Pac J Cancer Prev, 14, 2739-42.

Michalkiewicz E, Sandrini R, Figueiredo B, et al (2004). Clinical and outcome characteristics of children with adrenocortical tumors: a report from the International Pediatric Adrenocortical Tumor Registry. J Clin Oncol, 22, 838-45.

Missaoui N, Khouzemi M, Landolsi H, Jaidene L, et al (2011). Childhood Cancer Frequency in the Center of Tunisia. Asian Pac J Cancer Prev, 12, 537-42.

O’Neill CJ, Spence A, Logan B, et al (2010) Adrenal incidentalomas: risk of adrenocortical carcinoma and clinical outcomes. J Surg Oncol, 102, 450-3

Omura M, Saito J, Yamaguchi K, Kakuta Y, Nishikawa T (2004). Prospective study on the prevalence of secondary hypertension among hypertensive patients visiting a general outpatient clinic in Japan. Hypertens Res, 27, 193-202.

Pędziwiatr M, Natkaniec M, Kisialeuski M, et al (2014) .Adrenal incidentalomas: should we operate on small tumors in the era of laparoscopy?. Int J Endocrinol, 2014 .

Sadler C, Goldfarb M (2014). Risk estimator for adrenal tumor functionality. World J Surg, 38, 2019-24.

Sebastiano C, Zhao X, Deng FM, Das K (2013). Cystic lesions of the adrenal gland: our experience over the last 20 years. Hum Pathol, 44, 1797-803.

Stojadinovic A, Ghossein RA, Hoos A, et al (2002) .Adrenocortical carcinoma: Clinical, morphologic and molecular characterization. J Clin Oncol, 20, 941-950.

Treska V, Wirthová M, Hadravská S, Mukensnábl P, Kuntscher 
Shokouh Taghipour Zahir et al

V, Kreuzberg B, et al (2006) [Giant bilateral adrenal myelolipoma associated with congenital adrenal hyperplasia]. Zentralbl Chir, 131, 80-3.

Zhang J, Wang C, Gao J, et al (2014). Adrenal cortical neoplasms: a study of clinicopathological features related to epidermal growth factor receptor gene status. Diagn Pathol, 23, 19.

ZHANG Zhen-huan, LIU De-chun (2008).Clinical and pathological analysis of 62 cases of adrenal masses. Journal of Huaihai Medicine, China, 2008-05 